\title{
Macroglobulinemia de Waldenströn: comunicación de un caso
}

\author{
Waldenström's macroglobulinemia: report of a case
}

\author{
Franco Romaní ${ }^{1}$, José Cuadra ${ }^{2}$, Fernando Atencia ${ }^{3}$, Carlos Canelo ${ }^{2}$ \\ ${ }^{1}$ Interno de la Facultad de Medicina Humana, Universidad Nacional Mayor de San Marcos. Lima, Perú. \\ ${ }^{2}$ Unidad de Post Grado, Facultad de Medicina Humana, Universidad Nacional Mayor de San Marcos. Lima, Perú. \\ ${ }^{3}$ Docente de la Facultad de Medicina Humana, Universidad Nacional Mayor de San Marcos. Lima, Perú. \\ ${ }^{3}$ Hospital Nacional Arzobispo Loayza. Lima, Perú.
}

\begin{abstract}
Resumen
La macroglobulinemia de Waldenström (MW) es una rara neoplasia hematológica, caracterizada por la proliferación linfoplasmocitaria, con producción monoclonal de inmunoglobulina $\mathrm{M}(\mathrm{lg} \mathrm{M})$ e infiltración medular. Comunicamos el caso de un paciente de raza negra, con MW, quien presentó compromiso oftalmológico, con manifestaciones fundoscópicas, neurológicas, citopenia y síntomas constitucionales, derivados de la infiltración tumoral y de la gamapatia monoclonal. Asimismo, lesiones osteolíticas, raras en la MW. Se realiza una revisión de la literatura acerca de los criterios diagnósticos, epidemiología, fisiopatología y clínica de esta enfermedad muy poco informada en nuestro país.

Palabras clave: Macroglobulinemia de Waldenström; neoplasias; viscosidad; paraproteinemias.
\end{abstract}

\begin{abstract}
Waldenström's macroglobulinaemia (WM) is a rare haematological neoplasm characterized by bone marrow infiltration of lymphoplasmatic lymphoma and IgM monoclonal gammopathy. We report a case of a black patient with WM that presented ocular manifestations with funduscopic findings, neurologic manifestations, cytopenia, and constitutional symptoms related to tumor infiltration and monoclonal gammopathy. Also osteolytic bone lesions, rare in MW. We review the literature on the diagnostic criteria, epidemiology, pathogenesis and clinical manifestations of this disorder, unusual in our country.
\end{abstract}

Key words: Waldenstrom macroglobulinemia; neoplasms; viscosity; paraproteinemias.

\section{INTRODUCCIÓN}

La macroglobulinemia de Waldenström $(\mathrm{MW})$ es un trastorno proliferativo linfoplasmocitario maligno crónico, infrecuente, caracterizado por infiltración de médula ósea y producción monoclonal de $\operatorname{IgM}{ }^{(1-7)}$. Fue descrita hace 63 años por Jan Gosta Waldenström, quien informó sobre dos pacientes con sangrado oronasal, anemia, trombocitopenia, velocidad de sedimentación globular elevada, linfoadenopatías, viscosidad sérica alta, radiografías óseas normales y médula ósea con infiltrado linfoide. Estas observaciones preliminares probaron ser características distintivas del ampliamente reconocido, pero relativamente infrecuente, diagnóstico de MW ${ }^{(1,2,7)}$.

Se cree que la MW es predominantemente una enfermedad esporádica. Su causa es desconocida y no se puede descartar el posible rol de un defecto genético. Se ha visto que en pacientes con historia familiar de MW o de enfermedad de células plasmáticas el diagnóstico es más temprano y con mayor compromiso de la médula ósea. La predisposición familiar puede existir hasta en 20\% de pacientes con MW. Se ha confirmado deleciones en el cromosoma 6q21-22.1, en la mayoría de pacientes con MW sin historia familiar ${ }^{(1,3,8)}$.
El principal factor de riesgo para el desarrollo de MW es la preexistencia de gamapatia monoclonal IgM de significado incierto, que aumenta el riesgo relativo 46 veces respecto a la población general ${ }^{(1,8)}$. Se le ha asociado con el virus de la hepatitis $\mathrm{C}$, aunque recientemente se ha negado la relación ${ }^{(9,10)}$; también, se ha comunicado relación con el virus herpes humano 8 , la cual no ha sido confirmada.

Informamos el caso de un paciente de raza negra, en la que esta enfermedad es aún menos frecuente ${ }^{(1)}$, que presentó una clínica florida, con síntomas derivados de infiltración tumoral y de la gamapatia monoclonal, incluyendo el síndrome de hiperviscosidad, además de mostrar lesiones osteolíticas, las cuales son raras en la MW.

\section{CASO CLÍNICO}

Paciente varón de 64 años, de raza negra, natural y procedente de Lima, quien ingresó al Hospital Nacional Arzobispo Loayza con cuadro clínico de 8 meses de evolución, consistente en disnea a moderados esfuerzos, pérdida progresiva de peso e hiporexia. Cuatro meses antes del ingreso inició visión borrosa en ambos ojos y dificultad para la visión de cerca y de lejos. Tres meses antes del ingreso fue hospitalizado por empeoramien- to de la disnea y debilidad generalizada, detectándole anemia severa (hemoglobina de $2,4 \mathrm{~g} / \mathrm{dL}$ ), leucopenia y trombocitopenia. El paciente permaneció hospitalizado por 12 días, recibiendo 3 unidades de sangre y 2 unidades de plaquetas; fue dado de alta con mejoría notable. Sin embargo, la disnea progresó de esfuerzos moderados a leves, con mareos, vértigo y una disminución total de peso, de 24 kilos, en los últimos 8 meses. Por estos síntomas, el paciente acudió al consultorio de hematología, donde decidieron su hospitalización, para estudio. El paciente negó fiebre, dolores articulares, óseos, náuseas, vómitos, diarreas y cualquier otra molestia.

Entre los hábitos nocivos, el paciente era fumador de un paquete de cigarrillos al día, durante 50 años. Entre los antecedentes patológicos, tenía tomografía axial computada compatible con fibrosis pulmonar y espirometría con patrón restrictivo. Negó hipertensión arterial, diabetes mellitus, tuberculosis, hepatitis, fracturas patológicas, accidentes, reacciones adversas medicamentosas, contacto con tuberculosis y cirugías previas. No presentaba antecedentes familiares de importancia.

Al ingreso, el paciente tenía frecuencia cardiaca de 102 latidos por minutos, 24 respiraciones por minuto, con presión arterial 
de 100/70 mmHg., peso $58 \mathrm{~kg}$, talla $183 \mathrm{~cm}$ e índice de masa corporal de 17. Paciente no mostraba fascie característica, estaba hidratado, adelgazado, lúcido y colaborador al examen. La piel era tibia, elástica, pálida, sin equimosis ni petequias. El tejido celular subcutáneo estaba disminuido, no había edemas. No se palpó adenomegalias en las regiones cervical, axilar o inguinal. El cráneo era normocéfalo, conjuntivas pálidas, pupilas CIRLA (pupilas céntricas, isocóricas, reactivas a la luz y la acomodación). En el fondo de ojo, se observó en retina múltiples manchas hemorrágicas, en ojo derecho, papila con bordes netos y vasos anormales, venas aumentadas de calibre, tortuosas y cruces arteriovenosos patológicos. Las uñas eran hipocráticas (vidrio de reloj), lecho ungueal pálido. En la auscultación pulmonar se encontró crépitos secos bilaterales difusos, con murmullo que pasaba en ambos hemitórax. En el aparato cardiovascular, los ruidos cardiacos fueron rítmicos, de buena intensidad, sin soplos. El abdomen era blando, depresible, con ruidos hidroaéreos presentes, sin visceromegalia. No se encontró compromiso articular, esquelético, ni muscular. El examen neurológico fue normal.

Entre los exámenes auxiliares, se encontró hiperuricemia, inversión globulina/albúmina marcada, elevación de la velocidad de sedimentación y proteína $\mathrm{C}$ reactiva, y pancitopenia (Tabla 1 ). Se

Tabla 1. Exámenes auxiliares de ingreso.

\begin{tabular}{lc}
\hline Hemograma completo & Resultado \\
Hemoglobina & $4,4 \mathrm{gr} / \mathrm{dL}$ \\
Hematocrito & $14,6 \%$ \\
Plaquetas & $68000 / \mu \mathrm{L}$ \\
Leucocitos & $4140 / \mu \mathrm{L}$ \\
Neutrófilos & $50 \%$ \\
Segmentados & $48 \%$ \\
Abastonados & $2 \%$ \\
$\quad$ Linfocitos & $48 \%$ \\
Monocitos & $2 \%$ \\
Ácido úrico (VN: $4,3-8 \mathrm{mg} / \mathrm{dL})$ & $10,76 \mathrm{mg} / \mathrm{dL}$ \\
Calcio (VN: 8,6 - $10,5 \mathrm{mg} / \mathrm{dL})$ & $10,28 \mathrm{mg} / \mathrm{dL}$ \\
Creatinina & $2,4 \mathrm{mg} / \mathrm{dL}$ \\
Proteínas totales & $13,54 \mathrm{~g} / \mathrm{dL}$ \\
Albúmina & $2,71 \mathrm{~g} / \mathrm{dL}$ \\
Globulina & $10,83 \mathrm{~g} / \mathrm{dL}$ \\
Velocidad de sedimentación glomerular & $77 \mathrm{~mm} / \mathrm{hora}$ \\
Proteína C reactiva & $20,07 \mathrm{mg} / \mathrm{dL}$ \\
\hline
\end{tabular}

realizó aspirado de médula ósea y biopsia de hueso de espina ilíaca posterosuperior izquierda. En el aspirado de médula ósea se observó serie eritroide y mieloide, con actividad disminuida y detención en la maduración, linfocitos incrementados, con células plasmáticas inmaduras. Es decir, una médula ósea hipercelular para la edad, con predominio de linfocitos e incremento de

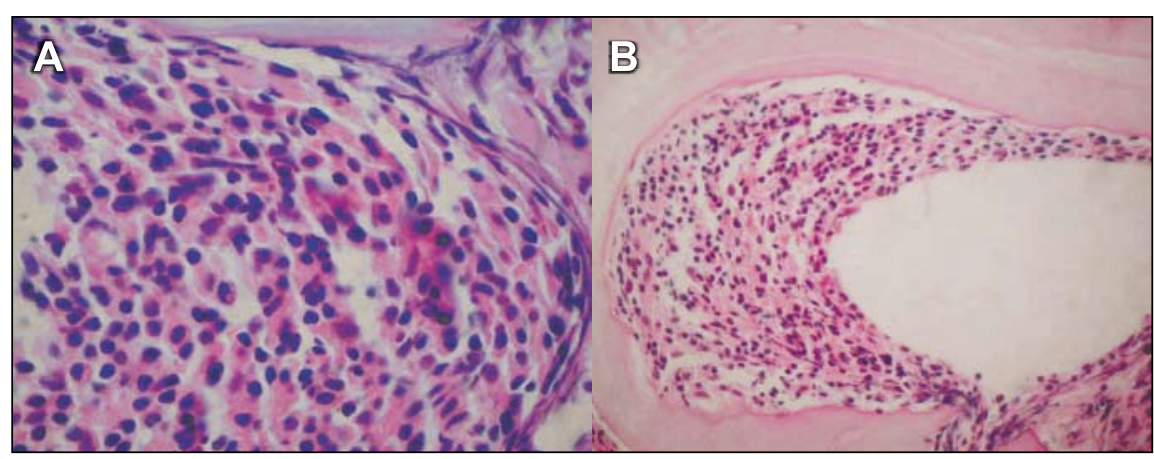

Figura 1. Biopsia de Médula osea (HE): Médula hipercelular con proliferación de células plasmáticas, con patrón intratrabecular. Los elementos de la serie eritroide y mieloide se encuentran disminuidos. (A: 400x, B: 100x).

células plasmáticas de aspecto inmaduro. La biopsia de médula ósea mostró una médula hipercelular, proliferación de células redondas con regular cantidad de citoplasma eosinófilo, núcleos de cromatina grumosa periférica, correspondientes a células plasmáticas que comprometían 80 a 90\% del tejido estudiado, patrón intratrabecular (Figura 1).

Entre los exámenes imagenológicos, en las placas de rayos X se observó huesos del cráneo, costillas y escápulas con alteraciones de la textura ósea e imágenes en sacabocados, difusas (Figura 2).

Se le realizó la electroinmunoforesis, obteniéndose IgM 3832 mg/dL, siendo el valor normal para la edad de 40 a $320 \mathrm{mg} / \mathrm{dL}$ (Figura 3).

Durante la hospitalización, el paciente desarrolló debilidad de miembros inferiores. Se le realizó electromiografía, en la que se encontró polineuropatía predominantemente motora distal crónica, asimétrica. Por problema de visión borrosa y en busca de signos y síntomas de síndrome de hiperviscosidad, se le realizó una retinografía, en la que se observó venas retinales dilatadas, con múltiples hemorragias retinales (Figura 4). Luego de establecido el diagnóstico, el paciente solicitó retiro voluntario, para manejo especializado en la seguridad social.

\section{DISCUSIÓN}

La macroglobulinemia de Waldenström (MW) es un trastorno proliferativo linfoplasmocitario maligno crónico, infrecuente, caracterizado por infiltración de médula ósea y producción monoclonal de IgM $(1-6,8)$.

La MW es una patología rara, tiene una incidencia total aproximada de 3 por millón de personas por año ${ }^{(1,8,11)}$, representa el $2 \%$ de todas las malignidades hematológicas en población caucásica ${ }^{(8)}$ y solo el $6 \%$ de las enfermedades linfoproliferativas de células $\mathrm{B}^{(11)}$. La incidencia es mayor y casi tres veces más frecuente en blancos ${ }^{(12)}$; solo $5 \%$ de pacientes con MW son de raza negra ${ }^{(1)}$. No se conoce las razones de estas diferencias en incidencia, pero reflejarían factores genéticos y ambiéntales ${ }^{(6,8)}$. Comparado con el mieloma múltiple $(\mathrm{MM})$ y la leucemia linfocítica crónica (LLC), la MW es rara, siendo la incidencia de LLC 3,8 por 100000 personas por año y la de MM, 5,5 por 100000 personas por año ${ }^{(8)}$.

En un estudio de 17 años realizado en México, en un centro especializado de hematología, en 7373 pacientes se encontró 11 casos de macroglobulinemia de Waldenström, con una media de edad de 65 años. En este estudio, 9 (82\%) pacientes presentaron anemia, al diagnóstico, 4 (36\%)

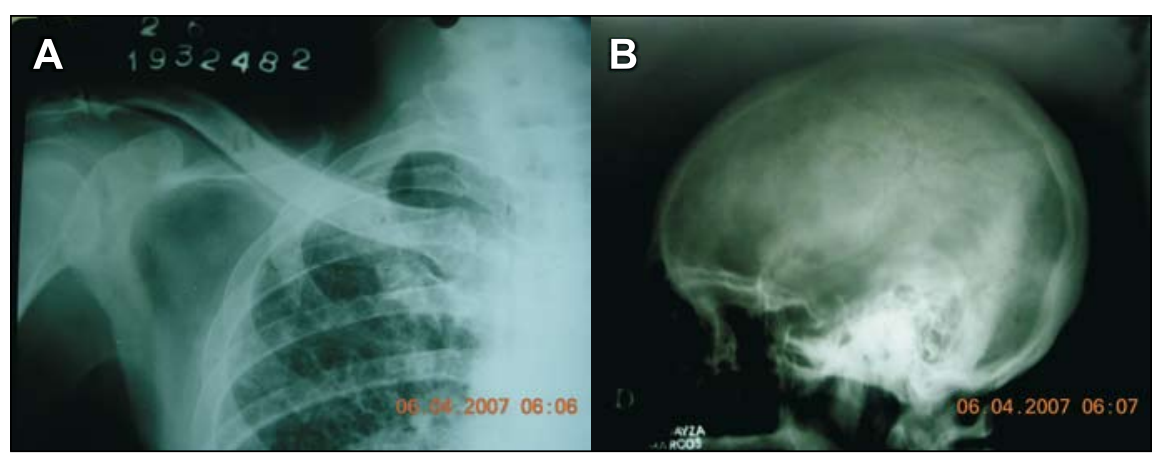

Figura 2. Radiografía simple de parrilla costal y escápula (A) con alteraciones de la textura ósea e imágenes en sacabocados difusas, las mismas alteraciones se observan en los huesos del cráneo (B). 


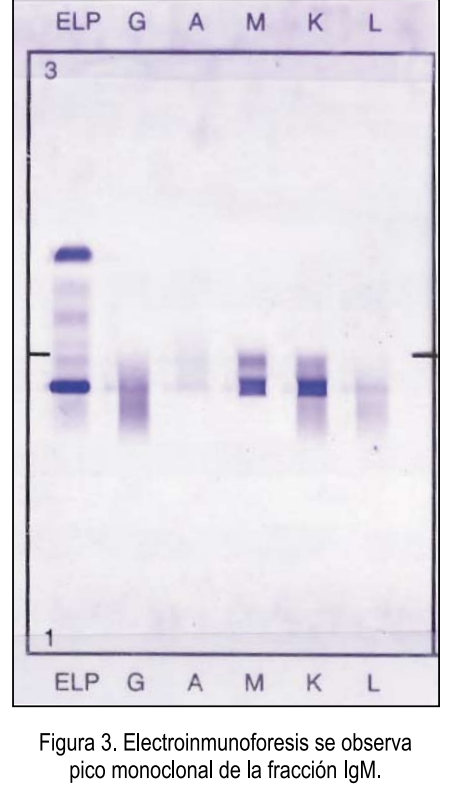

leucopenia y 3 (27\%) plaquetopenia. En México, representa 0,18\% de las malignidades hematológicas, siendo 11 veces menos frecuente que en población caucásica ${ }^{(6)}$. Phekoo y col. encontraron en Inglaterra 152 casos, entre 1999 y 2001; de ellos, 65\% tuvo más de 70 años, con una media de edad de 75 años. Obtuvieron una incidencia promedio de 0,55 por 100000 habitantes, siendo el grupo etáreo de 75 a 79 años el de mayor incidencia por edad, de 5,38 por 100 000 habitantes ${ }^{(11)}$. En España, Garcia-Sanz y col. encontraron 217 casos, entre 1989 y 1999, con una incidencia estimada para varones de 4,2 casos/millón personas año y de 2 casos/millón personas año para mujeres (12). En nuestra búsqueda bibliográfica, no encontramos datos estadísticos nacionales, debido a la poca información epidemiológica de esta enfermedad, a nivel nacional y latinoamericano; la mayoría consiste en comunicaciones de casos o series basadas en pocos pacientes.

Los criterios diagnósticos de MW fueron definidos durante el Segundo Taller

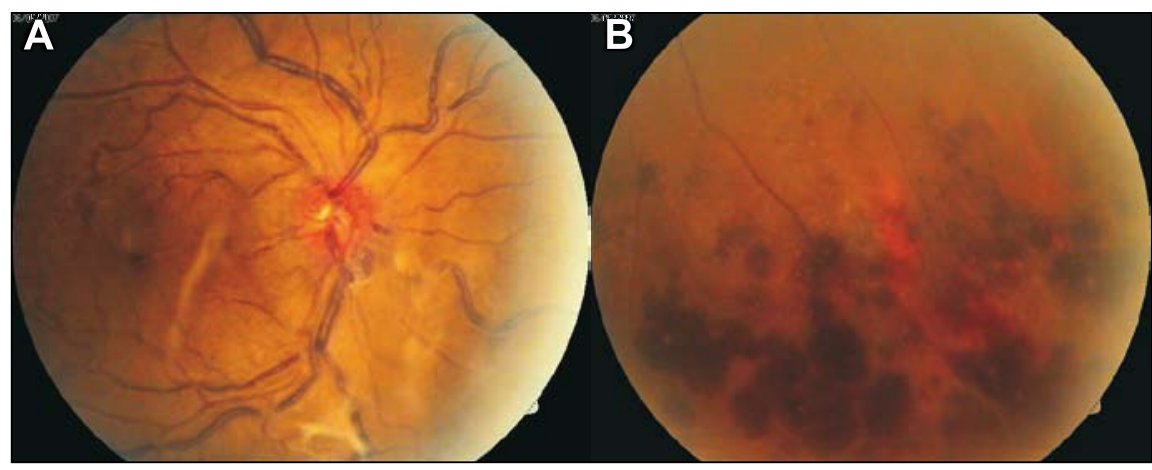

Figura 4. Retinografía muestra dilatación, segmentación y tortuosidad venosa (A), además Hemorragias retinianas múltiples y presencia de exudado algodonoso (B).
Internacional sobre la Macroglobulinemia de Waldenström, en setiembre 2002, en Atenas ${ }^{(1-3)}$, y son los siguientes:

- Proteínas monoclonales IgM de cualquier concentración.

- Infiltración en médula ósea por pequeños linfocitos, que muestran diferenciación plasmocitoide/células plasmáticas.

- Patrón intertrabecular de infiltración de médula ósea.

- IgM de superficie: inmunofenotipo CD5 \pm , CD10-, CD19+, CD20+, $\mathrm{CD} 22+, \mathrm{CD} 23-, \mathrm{CD} 25+, \mathrm{CD} 27+$, FMC7+, CD103-, CD 138

Se sugiere que la MW debería ser considerada como una entidad clínico patológica distinta y ser sospechada como fuerte posibilidad diagnóstica en aquellos pacientes con linfoma linfoplasmocitoide que tienen gamapatia monoclonal IgM. Sin embargo, la demostración de una gamapatia monoclonal IgM no es sinónimo de MW, ya que esta anormalidad puede ser vista en muchas formas de enfermedad linfoproliferativa de células B, como en la gamapatia monoclonal de significado incierto (MGUS) ${ }^{(1,3)}$.

En cuanto al corte mínimo de IgM sérica, el valor de $3 \mathrm{~g} / \mathrm{dL}$ fue usado para definir MW de otros linfomas de células B de grado bajo o de gamapatia monoclonal de significado incierto. Sin embargo, no hay consenso sobre qué valor de IgM es confiable para diferenciar MW de otros tipo de linfomas. La clasificación franco-americana recomienda $2 \mathrm{~g} / \mathrm{dL}$; otros sugieren puntos de corte de 1 a $3 \mathrm{~g} / \mathrm{dL}$. Parece que los niveles de IgM mayores de $3 \mathrm{~g} / \mathrm{dL}$ favorecen fuertemente el diagnóstico de MW. Pero, valores menores no excluyen el diagnóstico ${ }^{(2)}$. Por eso, el Segundo Taller Internacional sobre la Macroglobulinemia de Waldenström propone una proteína monoclonal IgM elevada, en cualquier concentración, sin un punto de corte $^{(1-5)}$
Los términos usados para designar los linfomas asociados con la MW en diferentes sistemas de clasificación han evolucionado en las últimas décadas; estos términos incluyen ${ }^{(2)}$ :

- Linfoma plasmocitoide linfocítico bien diferenciado (clasificación de Rappaport).

- Linfoma linfocítico-plasmocítico (clasificación de Lukes-Collins).

- Inmunocitoma (clasificación de Kiel).

- Linfoma de linfocitos pequeños con diferenciación plasmocitoide (clasificación de la Working Formulation).

- Linfoma linfoplasmocitoide/inmunocitoma (clasificación REAL).

- Linfoma linfoplasmocítico (clasificación de la OMS).

Existe una clasificación de MW y trastornos relacionados, planteados en el Segundo Taller Internacional sobre la Macroglobulinemia de Waldenström, en Atenas, y son los siguientes ${ }^{(1,3-5)}$ :

- MW sintomática: cuando hay proteína IgM monoclonal infiltrada en médula ósea, síntomas por IgM y síntomas de infiltración tumoral.

- MW asintomática: existen proteínas monoclonales e infiltrado de medula ósea, pero no síntomas por IgM o de infiltración tumoral.

- Trastornos relacionados con paraproteinemia IgM: existe proteína monoclonal IgM, con síntomas derivados de la proteína monoclonal IgM, pero sin evidencias de linfoma o infiltración medular.

- Gamapatia monoclonal de significado incierto (GMSI IgM): solo existe proteína IgM monoclonal. No hay síntomas derivados, ni infiltración en médula ósea.

En el presente caso, el paciente tuvo MW sintomática, con síntomas derivados de infiltración tumoral y de la gamapatia monoclonal.

Por la infiltración tumoral, se pueden producir citopenia, fiebre, sudoración nocturna, pérdida de peso, linfadenopatias y organomegalia. Mientras que, por la gamapatia monoclonal se producen síndrome de hiperviscosidad, crioglobulinemia, neuropatía, amiloidosis y aglutininas frías ${ }^{(1-4)}$.

La linfadenopatia ocurre en 15 a 20\% de pacientes al momento de la presentación y usualmente no es tan prominente como la encontrada en pacientes con otros tipos de linfoma no-Hodgkin. La esplenomegalia ocurre en 10 a 20\% de pacientes. La diseminación a bazo, piel, pulmón y tracto 
gastrointestinal muestra similar patrón de infiltración que en la médula ósea o en ganglios linfáticos ${ }^{(2,13)}$.

Muchas de las manifestaciones de la MW son resultado de las propiedades de la IgM monoclonal, incluyendo su gran tamaño y su alto contenido de carbohidratos, con moléculas asimétricas grandes, el 80\% intravascular. Una concentración alta de estas proteínas resulta en incremento de la presión osmótica, por su componente carbohidratado, que atrae agua, y produce incremento de la resistencia al flujo y daño del tránsito a través de la microcirculación. Se producen síntomas y signos de hiperviscosidad, los cuales se agravan por la interacción de la IgM con los eritrocitos, incrementando la viscosidad interna y reduciendo la deformabilidad de los glóbulos rojos ${ }^{(1,3,5)}$. Existe crioglobulinemia clínicamente evidente, con fenómeno de Raynaud, úlceras dérmicas, necrosis y urticaria fría en menos de $5 \%$ de pacientes ${ }^{(1,3)}$. La IgM monoclonal puede interactuar con proteínas circulantes, incluyendo factores de coagulación, y pueden dañar la adhesión y agregación plaquetaria, con prolongación en el tiempo de coagulación y de sangría, respectivamente. Las proteínas monoclonales pueden ser depositadas en muchos tejidos, las macroglobulinas pueden quedar atrapadas en las asas glomerulares, pueden precipitar y formar depósitos subendoteliales, que producen daño glomerular, con proteinuria no selectiva, deshidratación y uremia ${ }^{(3)}$. Se ha informado las pápulas y nódulos dérmicos como macroglobulinemia cutis $^{(1,3)}$. En los casos ocasionales de diarrea, malabsorción o sangrado gastrointestinal, la biopsia mostró depósito de IgM en la lámina propia y submucosa del intestino (14). El compromiso gastrointestinal de la MW es raro y se da por varios mecanismos; el modo más común es el compromiso vía depósito de IgM de cadena ligera, visible en la tinción rojo congo. Otro mecanismo es la infiltración de células linfoplasmocíticas en la pared del tracto gastrointestinal, con producción local de IgM en la lámina propia, la cual es captada por los canales linfáticos. Sin embargo, la alta viscosidad del fluido intersticial puede producir dilatación linfática y obstrucción, con linfangiectasia secundaria. La manifestación clínica de la linfagiectasia intestinal es una enteropatía perdedora de proteínas, con diarrea y síndrome de malabsorción ${ }^{(15)}$.

La amiloidosis primaria, por depósito de cadenas ligeras monoclonales, ocurre principalmente en el corazón, nervio periférico, riñones, tejidos blandos, hígado y pulmones (en orden de frecuencia) ${ }^{(1,3)}$.

Existen manifestaciones relacionas a la actividad anticuerpo de la IgM, que actúan como anticuerpos contra antígenos autólogos. Hasta 20\% de pacientes presenta neuropatía periférica relacionada a IgM, la entidad más común es una neuropatía periférica desmielinizante crónica, simétrica y distal, en que la IgM actúa contra el componente glicoproteico del nervio ${ }^{(1,3)}$. En ocasiones, la IgM monoclonal puede actuar como anticuerpo contra la membrana basal del glomérulo, piel y retina; como consecuencia se produce glomerulonefritis, pénfigo paraneoplásico y retinitis ${ }^{(3)}$.

Aproximadamente $30 \%$ de todos los pacientes con MW desarrolla complicaciones coriorretinales. Las complicaciones oculares, como en el caso presentado, son más comúnmente el resultado indirecto de la hiperviscosidad sérica. La microvasculatura de la retina y coroides son particularmente susceptibles a los efectos de la hiperviscosidad. Como la concentración de IgM circulante se incrementa, hay disminución de flujo sanguíneo a la retina. Este daño circulatorio resulta en incremento sustancial en la presión intravascular de la circulación venosa retiniana y produce los hallazgos clínicos de obstrucción venosa retiniana. La presencia de retinopatía hemorrágica con venas retinales tortuosas y dilatadas es comúnmente llamado fondo disproteinémico, lo cual sugiere una forma de presentación más agresiva de MW. Aunque los hallazgos fundoscópicos en MW no son patognomónicos, ellos pueden ser la primera clave para el diagnóstico ${ }^{(1,7,16)}$. Es un error considerar que los pacientes con MW se encuentran frecuentemente afectados por el síndrome de hiperviscosidad; la frecuencia de este síndrome en MW varía de 6 a 20\%, dependiendo de las series. Actualmente, este síndrome es infrecuente, probablemente debido al diagnóstico temprano y a la plasmaféresis profiláctica de pacientes de riesgo alto ${ }^{(2,7)}$.

Las lesiones osteolíticas son raras en la MW, siendo frecuentes en el mieloma múltiple. En el caso que presentamos, tanto en la placa de cráneo como a nivel de la parrilla costal se observó lesiones en sababocados, lo cual no es frecuente en MW, ya que la asociación de gamapatia IgM con lesiones osteolíticas difusas es rara ${ }^{(7)}$, aproximadamente en solo $2 \%$ de casos de MW ${ }^{(13)}$. Esta particularidad, en el caso del paciente, podría explicarse por: transformación de la enfermedad en linfoma de células grandes, la presencia de MW osteolítico, mieloma múltiple como segunda enfermedad asociada y por transformación clonal de MW a mieloma múltiple; existe un caso publicado de esta evolución ${ }^{(13)}$.

La anemia es multifactorial; se debe a la expansión clonal en la medula ósea, al incremento del volumen plasmático e hiperviscosidad y por niveles elevados de IL6. La trombocitopenia se ve generalmente solo en casos con compromiso extenso de la medula ósea por las células clonales de la MW ${ }^{(13)}$, como ocurrió con este paciente.

Dentro del esquema de trabajo para confirmar un diagnóstico de MW ${ }^{(1-4)}$ se tiene:

1. Electroforesis de proteínas séricas.

2. Inmunofijación. Para caracterizar el tipo de cadenas pesadas o ligeras.

3. Electroforesis en orina de 24 horas; 40 a $80 \%$ tiene proteinuria de Bences-Jones detectable.

4. $\beta 2$-microglobulina sérica, evaluación del pronóstico.

5. Biopsia de médula ósea, infiltrado linfoplasmocitico intratrabecular, que va desde predominio de células linfociticas o linfoplasmocíticas hasta células plasmáticas.

6. Estudios citogenéticos, opcional.

7. Tomografía computada de abdomen y pelvis, para detectar organomegalia y linfadenopatías (el survey esquelético y el scan óseo no son necesarios, en ausencia de síntomas, ya que las lesiones líticas óseas son inusuales y más frecuentes en el mieloma múltiple)

8. Viscosidad sérica o sanguínea, si los signos y síntomas de síndrome de hiperviscosidad están presentes o la IgM es mayor de 5000.

En el estudio de nuestro paciente, se realizó el estudio de médula ósea, con el aspirado y biopsia, encontrándose un infiltrado linfoplasmocitario, a predominio plasmático, con patrón intratrabecular. En la electroforesis de proteínas séricas se encontró la IgM en 3832 mg/dL, obteniéndose tres de los cuatro criterios diagnósticos de MW.

Desde el 2002, cuando se realizó el Segundo Taller Internacional en Grecia y se dieron los criterios diagnósticos para MW, se han desarrollado dos talleres. En Francia (2004), se realizó el Tercer Taller Internacional sobre MW y, en el año 2007, en Kos (Grecia), el Cuarto taller. Este año se realizará el Quinto Taller Internacional de MW, en Estocolmo. En el 2004 (Francia), se reafirmó la recomendación de consenso 
previo de que el tratamiento está reservado para pacientes sintomáticos y no debería ser iniciado solo en base al nivel de proteínas monoclonal sérico ${ }^{(1,17)}$. Las indicaciones para iniciar el tratamiento son: hemoglobina menor de $10 \mathrm{~g} / \mathrm{dL}$, plaquetas menos de $100 / \mu \mathrm{L}$, adenopatías y organomegalia significante, síndrome de hiperviscosidad, neuropatía severa, amiloidosis, crioglobulinemia, enfermedad de aglutininas frías o evidencia de transformación de enfermedad (1-5,7,17). Sin embargo, niveles de más de 50 $\mathrm{g} / \mathrm{L}$ ponen al paciente en un riesgo alto de hiperviscosidad. En este caso, se requiere historia clínica y examen físico completo y examen fundoscópico, para encontrar síntomas y signos tempranos de hiperviscosidad y evitar que el tratamiento sea pospuesto $^{(1,17)}$.

Las principales opciones en la terapia de primera línea son los agentes alquilantes (cloranbucilo, ciclofosfamida, melfalán), análogos de purina (cladribina, fludarabina) y anticuerpos monoclonales (rituximab). La plasmaféresis es indicada para manejo agudo de pacientes con síntomas de hiperviscosidad $^{(1-5,7,17)}$.

En conclusión, la MW es una neoplasia linfoplasmocitaria rara, caracterizada por sobreproducción monoclonal de IgM e infiltración medular, mecanismos de los cuales provienen todos los síntomas y signos atribuibles a la enfermedad y que en nuestro paciente se presentaron floridamente. Esto permitió realizar una revisión, definir conceptos sobre diagnóstico, epidemiología, patogénesis de síntomas y signos de esta patología. Además, la poca literatura existente a nivel nacional sobre $\mathrm{MW}$, hace importante su informe y discusión.

\section{REFERENCIAS BIBLIOGRÁFICAS}

1. Vijay $A$, Gertz M. Waldenström macroglobulinemia. Blood. 2007;109(12):5096-103.

2. Lin P, Medeiros LJ. Lymphoplasmacytic lymphoma/ Waldenstrom macroglobulinemia: an evolving concept. Adv Anat Pathol. 2005;12:246-55.

3. Dimopoulos MA, Kyle RA, Anagnostopoulos A, Treon SP. Diagnosis and management of Waldenstrom's macroglobulinemia. J Clin Oncol. 2005;23(7):1564-77.

4. García-Sanz R, González. M, Ocio E. Macroglobulinemia de Waldenström. Haematologica. 2004;89:89-104.

5. Khosravi P, Del Castillo A, Díaz VM. Macroglobulinemia de Waldenström. An Med Interna. 2006;23(6):291-3.

6. Ruiz-Argüelles GJ, Ramírez-Cisneros FJ, FloresMartínez J, CernudaGraham MC. Waldenströjm's macroglobulinemia is infrequent in Mexican mestizos: Experience of a hematological diseases referral center. Rev Invest Clin Mex. 2000;52:4979.

7. Fonseca R, Hayman S. Waldenström's macroglobulinaemia. British Journal of Haematology. 2007;138:700-20.

8. McMaster M, Neil Caporaso N. Waldenström's macroglobulinaemia and $\mathrm{IgM}$ monoclonal gammopathy of undetermined significance: emerging understanding of a potential precursor condition. British Journal of Haematology. 2007;139(5):663-71.

9. Silvestri F, Barillari G, Fanin R. Risk of hepatitis C virus infection, Waldenstrom's macroglobulinemia, and monoclonal gammopathies. Blood. 1996;88:1125-6.

10. Leleu X, O'Connor K, Ho AW, Santos DD, Manning $\mathrm{R}, \mathrm{Xu} \mathrm{L}$, et al. Hepatitis $C$ viral infection is not associated with Waldenström's macroglobulinemia. Am J Hematol. 2007;82(1):83-4.

11. Phekoo KJ, Jack RH, Davies E, Møller H, Schey SA, On behalf of consultant haematologists, South Thames Haematology Specialist Committee.
The incidence and survival of Waldenstrom's Macroglobulinaemia in South East England. Leukemia Research. 2008;32:55-9.

12. García-Sanz R, Montoto S. Waldenström `s macroglobulinaemia: presenting features and outcome in a series with 217 cases. British Journal of Haematology. 2001;115:575-82.

13. Jondeau K, Alterescu R, Franc B, Davi F, Massé $J$, Boukour $S$, et al. Unusual evolution of Waldenström `s macroglobulinemia into osteolytic myeloma. European Journal of Haematology. 2006;77(1):74-9.

14. Case Records of the Massachusetts General Hospital: Weekly clinicopathological exercises: Case 3-1990-A 66-year-old woman with Waldenström's macroglobulinemia, diarrhea, anemia and persistent gastrointestinal bleeding. N Engl J Med. 1990;322:183-92.

15. Pratz KW, Dingli D, Smyrk TC, Lust JA. Intestinal lymphangiectasia with protein-losing enteropathy in Waldenström 's macroglobulinemia. Medicine. 2007;86:210-4.

16. Pilon AF, Rhee PS, Messner LV. Bilateral, persistent serous macular detachments with Waldenström 's macroglobulinemia. Optometry and Vision Science. 2005;82(7):573-8.

17. TreonSP, Gertz MA, Dimopoulos M, Anagnostopoulos A, Blade J, Branagan AR, et al. Update on treatment recommendations from the Third International Workshop on Waldenstrom's macroglobulinemia. Blood. 2006;107:3442-6.

Manuscrito recibido el 10 de marzo de 2008 y aceptado para publicación el 13 de mayo de 2008.

Correspondencia:

Franco Romaní Romaní

Av. Héroes del Pacifico 615, Chorrillos.

Lima 09, Perú

Correo-e: frrr812@hotmail.com 